\title{
Aggregated LDL and Lipid Dispersions Induce Lysosomal Cholesterol Accumulation in Macrophage Foam Cells
}

\author{
W. G. Jerome, E. E. Griffin, B. E. Cox, and J. C. Ullery
}

Department of Pathology, Vanderbilt University Medical Center, $116121^{\text {st }}$ Ave, South, Nashville, TN 37232-2561

A hallmark feature of the atherosclerotic lesion is the presence of cholesterol engorged macrophages called foam cells because of the foamy appearance that lipid imparts to their cytoplasm ${ }^{1}$. Cytochemical inspection reveals that much of this cholesterol accumulates within large swollen lysosomes ${ }^{2}$. Although the cholesterol in the atherosclerotic lesion is primarily derived from LDL, it must be modified by the lesion environment before LDL can produce the massive cholesterol accumulation seen in atherosclerosis ${ }^{3}$. Oxidation of LDL is just such a modification and we have mimicked this lysosomal cholesterol accumulation by treating macrophages in culture with mildly oxidized LDL (ox-LDL). The cholesterol in LDL particles is primarily in the form of cholesteryl esters (CE). Following uptake by receptor mediated endocytosis, this $\mathrm{CE}$ is delivered to lysosomes/late endosomes for hydrolysis to free cholesterol (FC) and fatty acids ${ }^{2}$. The lysosomal cholesterol accumulation from ox-LDL occurs in two phases, an initial phase where lysosomal hydrolysis occurs normally but the resulting FC is trapped in lysosomes and a second phase where lysosomal CE hydrolysis is inhibited ${ }^{4}$. The result is an accumulation of both FC and CE in lysosomes. This cholesterol is trapped and not available for further metabolism by the cell, presenting grave consequences for the cell and potentially accelerating development of the atherosclerotic lesion.

Curiously, another modification of LDL, acetylation, does not produce lysosomal cholesterolengorgement, leading to speculation that it is the oxidized lipids which disrupt lysosomal hydrolysis 5. Ox-LDL is not the only modified form of cholesterol found in atherosclerotic lesions and so in the present study we investigate uptake and metabolism by macrophages of two non-oxidized modified lipids; small aggregates of LDL (agg-LDL) and CE-rich lipid dispersions (DISP) which were formed from sonicating together phosphatidylcholine, phosphatidylserine, and CE. Cellular cholesterol accumulation was analyzed by biochemical measures of FC and $\mathrm{CE}$ mass and the rate of CE hydrolysis and by fluorescence, brightfield, and electron microscopy.

Incubation of the human macrophage cell line, THP-1, with either agg-LDL (Figure 1) or DISP produced cellular cholesterol accumulation of greater than $300 \mathrm{ug}$ total cholesterol $/ \mathrm{mg}$ cell protein as both FC and CE. For the first two days of incubation, cells treated with agg-LDL continued to generate some FC via lysosomal hydrolysis although lysosomal $\mathrm{CE}$ also accumulated. However, after the second day, greater than $95 \%$ of the cholesterol accumulating in agg-LDL-treated cells occurred as unhydrolyzed lipoprotein in lysosomes. DISP treatment also produced both FC and CE accumulation but the rate of accumulation occurred faster and less FC was generated. The timing of the shutdown of hydrolysis appeared related to the rate of initial free cholesterol accumulation.

Measurement of lysosomal $\mathrm{pH}$ revealed that the cholesterol engorged lysosomes failed to maintain an acidic $\mathrm{pH}$, suggesting that the shutdown of hydrolysis was related to failure to maintain the proper lysosomal $\mathrm{pH}$. This failure to maintain an acid $\mathrm{pH}$ was true for cells treated with ox-LDL, 
agg-LDL, or DISP and the timing of the inhibition of acidity roughly followed the timing of inhibition of hydrolysis. It also coincided with the rate of FC generation; with DISP treated cells showing lysosomal failure quicker than agg-LDL treatment and with ox-LDL taking the longest time.

If an alteration in the lysosomal $\mathrm{pH}$ is the explanation for cessation of lipolysis, one would expect proteolysis to also be inhibited. Using immunocytochemical staining for apolipoprotein $\mathrm{B}$, the major protein found in LDL and its modified varieties, we found that initially no stainable apolipoprotein B can be seen in cells treated with acetylated-LDL (ac-LDL) or agg-LDL. This indicates efficient breakdown of apolipoprotein B in lysosomes. However, with extended incubation, stainable apolipoprotein B accumulated in agg-LDL-treated but not ac-LDL-treated cells. Western blots of agg-LDL treated cells initially show no full length apolipoprotein B. However, coincident with lack of acidification, full length apolipoprotein B accumulates. This accumulation is not seen with acLDL treatment. .

These data show that oxidized lipids are not required for the inhibition of lysosomal CE hydrolysis. Moreover, they suggest that the initial FC accumulation in lysosomes may alter lysosomal membranes such that the acidic $\mathrm{pH}$ necessary for lysosomal function cannot occur. This leads to an overall lysosomal dysfunction which could have severe implications for the development of atherosclerotic lesion.

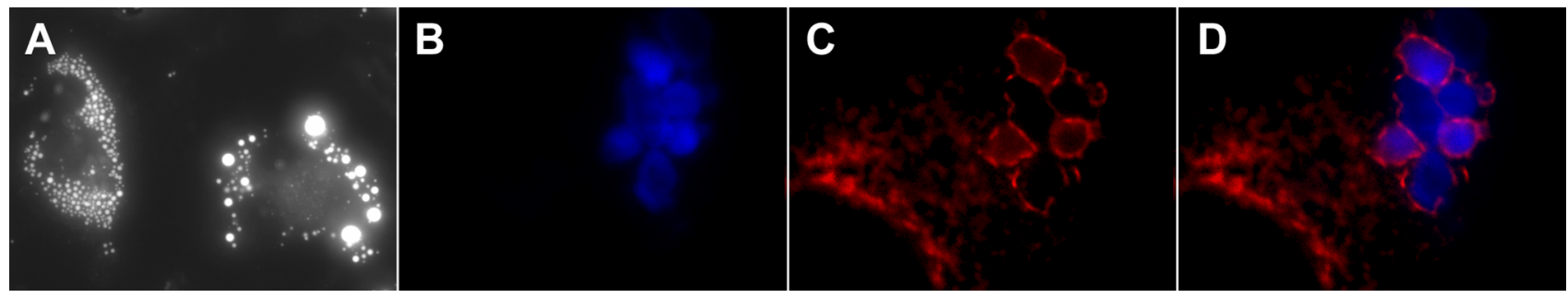

Figure 1: THP-1 cells incubated with agg-LDL for 6 days. A) Nile red staining for neutral lipid. B) Filipin staining for FC. C) LAMP-1 staining as marker for lysosome/late endosome. D) Localization of FC in LAMP-1 positive vesicles.

\section{References:}

1. St. Clair R. Pathogenesis of Atherosclerosis. Cardiol Rev. 1997;5:14-24.

2. Jerome WG, Yancey PG. The role of microscopy in understanding atherosclerotic lysosomal lipid metabolism. Microsc Microanal. 2003;9:54-67.

3. Steinberg D, Parthasarathy S, Carew T, Khoo J, Witztum J. Beyond cholesterol, modification of low-density lipoproteins that increase its atherogenicity. New Eng J Med. 1989;320:915924.

4. Yancey PG, Jerome WG. Lysosomal sequestration of free and esterified cholesterol from oxidized low density lipoprotein in macrophages of different species. J Lipid Res. 1998;39:1349-1361.

5. Hoff H, O'Neill J. Lesion-derived low density lipoprotein and oxidized low density lipoprotein share a lability for aggregation, leading to enhanced macrophage degradation. Arterioscler Thromb. 1991;11:1209-1222.

6. This work was supported by a grant 2 R01 HL49148-04A2 from NIH, NHLBI 